\title{
Elliptic CY3folds and non-perturbative modular transformation
}

\author{
Amer Iqbal ${ }^{1, a}$, Khurram Shabbir ${ }^{2, b}$ \\ ${ }^{1}$ Abdus Salam School of Mathematical Sciences, Government College University, Lahore, Pakistan \\ ${ }^{2}$ Department of Mathematics, Government College University, Lahore, Pakistan
}

Received: 10 February 2016 / Accepted: 7 March 2016 / Published online: 16 March 2016

(c) The Author(s) 2016. This article is published with open access at Springerlink.com

\begin{abstract}
We study the refined topological string partition function of a class of toric elliptically fibered Calabi-Yau threefolds. These Calabi-Yau threefolds give rise to five dimensional quiver gauge theories and are dual to configurations of M5-M2-branes. We determine the GopakumarVafa invariants for these threefolds and show that the genus $g$ free energy is given by the weight $2 \mathrm{~g}$ Eisenstein series. We also show that although the free energy at all genera are modular invariant, the full partition function satisfies the non-perturbative modular transformation property discussed by Lockhart and Vafa in arXiv:1210.5909 and therefore the modularity of free energy is up to non-perturbative corrections.
\end{abstract}

\section{Introduction}

In this paper we study the refined topological string partition function of a class of toric elliptically fibered Calabi-Yau threefolds which are dual to a set of parallel M5-branes with a transverse direction compactified to a circle. The size of the circle is related to the Kähler parameter of the elliptic curve class on the Calabi-Yau threefolds. We determine the Gopakumar-Vafa invariants for all curve classes and also show that for these Calabi-Yau threefolds the genus $g$ free energy takes a particularly simple for and is given by the Eisenstein series. We also study the modular properties of the refined partition function and show that it satisfies the nonperturbative modular transformation discovered in [1]. As was shown in [1] for the case of $N=1, N$ being the number of M5-branes, the partition function is not modular invariant but satisfies a more involved transformation which maps the $g_{s}$ to $\frac{1}{g_{s}}$ where $g_{s}$ is the topological string coupling constanthence the name non-perturbative modular transformation.

This note is organized as follows. In Sect. 2 we discuss the Calabi-Yau geometry and the dual brane configuration.

\footnotetext{
a e-mail: amer@alum.mit.edu

be-mail: khurramsms@gmail.com
}

In Sect. 3 we use the refined topological vertex formalism to express the partition function as a trace of an operator acting on the fermionic Fock space. In Sect. 4 we determine the Gopakumar-Vafa invariants for all curve classes and the genus $g$ fee energy. In Sect. 5 we express the partition function in terms of double elliptic gamma functions and show that it satisfies the non-perturbative modular transformation property.

\section{Elliptic CY3fold and dual brane configuration}

The class elliptic Calabi-Yau threefolds we are interested in are dual to certain brane configurations which arose in the study of M-strings [2]. These Calabi-Yau threefolds are birationally equivalent $\widehat{A}_{N-1} \times{ }_{f} \mathbb{C}$ where $\widehat{A}_{N-1}$ is affine $A_{N-1}$ space blown up at $N$ points and it is fibered over $\mathbb{C}$ to obtain the Calabi-Yau threefold. We will denote these by $X_{N}$.

The affine $A_{N-1}$ space has $N \mathbb{P}^{1}$ 's corresponding to the simple roots of affine $S U(N)$. We denote these curve classes by $C_{a}$ with $a=1,2, \ldots, N$. The blow-up introduces $N$ new curve classes, which we denote by $M_{a}$ with $a=1,2, \ldots, N$. The class of the elliptic curve $E$ is given by

$E=C_{1}+C_{2}+\cdots+C_{N}$.

The complexified Kähler parameters associated with these curves are given by the Kähler form $\omega$ :

$t_{a}=\int_{C_{a}} \omega, \quad \rho=\int_{E} \omega, \quad m=\int_{M_{a}} \omega, \quad a=1,2, \ldots, N$.

Because of Eq. (2.1) $\rho$ is given by

$\rho=t_{1}+t_{2}+\cdots+t_{N}$.

The $(p, q)$ 5-brane web dual to $X_{N}$ and the Newton polygon are given by Fig. 1a, b, respectively. 
Fig. 1 a The $(p, q)$ brane configuration dual to $X_{N}$. The web lives on $\mathbb{R} \times S^{1}$ and the horizontal lines are glued together. b The Newton polygon of $X_{N}$

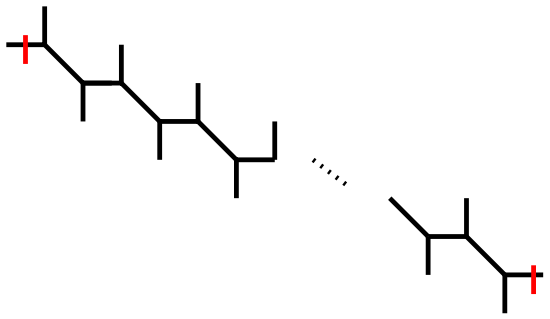

(a)

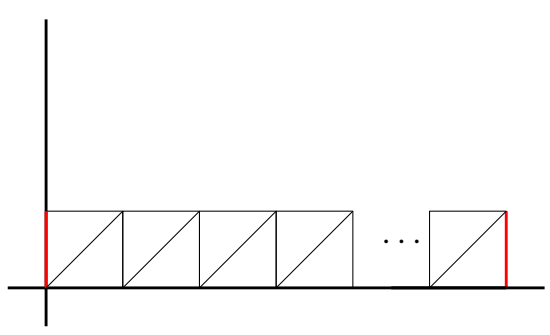

(b)
The Calabi-Yau threefolds $X_{N}$ are dual to certain M5brane configurations. Consider $N$ M5-brane with worldvolume coordinates $X^{A}, A=0,1,2,3,4,5$. The transverse space is $\mathbb{R}^{5}$ with coordinates $X^{I}, I=6,7,8,9,10$. We consider the $X^{6}$ direction and separate the $N$ M5-branes along this direction and then compactify this direction to a circle so that M5-branes are sprinkled on the $X^{6}$ circle. The transverse space to the M5-branes is $\mathbb{R}_{\perp}^{4} \times S^{1}$ and one can introduce a deformation by fibering this transverse space over the $X^{6}$ circle so that as one goes around the $X^{6}$ circle there is an $S O(4)$ action on $\mathbb{R}_{\perp}^{4}$ given by [2],

$\left(w_{1}, w_{2}\right) \mapsto\left(e^{2 \pi i m} w_{1}, e^{-2 \pi i m} w_{2}\right)$.

The deformation $m$ breaks the maximal supersymmetry of the brane configuration. In the type IIB picture the theory living on the $(p, q)$ brane web is five dimensional quiver gauge theory with eight supercharges. The partition function of this gauge theory can be calculated using Nekrasov's instanton calculus or by applying the refined topological vertex formalism to the $(p, q)$ brane web of Fig. 1a.

\section{The partition function from topological vertex}

The partition function of the $(p, q)$ brane web shown in Fig. 1 can be calculated by applying refined topological vertex formalism to the web. The refined vertex requires a choice of preferred direction [3] which determines the form of the partition function as a sum over Young diagrams but the partition function itself is invariant under the change in the preferred direction. In this case the simplest choice for the preferred direction is vertical and, as we will see below, in this case the sum can be carried out exactly and partition function becomes an infinite product. We denote the topological string partition function of this Calabi-Yau threefold by $Z_{N}$ and it is given by [4]

$$
\begin{aligned}
Z_{N}:= & \sum_{\vec{\lambda}} \prod_{a=0}^{N-1}\left[\left(-Q_{a}\right)^{\left|\lambda_{(a)}\right|}\right. \\
& \left.\times\left(\sum_{\mu}\left(-Q_{m}\right)^{|\mu|} C_{\lambda_{(a)}^{t} \mu \emptyset}(t, q) C_{\lambda_{(a+1)} \mu^{t} \emptyset}(q, t)\right)\right],
\end{aligned}
$$

where

$$
\begin{aligned}
C_{\lambda \mu \emptyset}(t, q)= & \left(\frac{q}{t}\right)^{\frac{|\lambda|-|\mu|}{2}} \\
& \times \sum_{\eta}\left(\frac{q}{t}\right)^{\frac{|\eta|}{2}} s_{\lambda^{t} / \eta}\left(t^{-\rho}\right) s_{\mu / \eta}\left(q^{-\rho}\right)
\end{aligned}
$$

is the refined topological vertex. The length of the slanted lines in Fig. 1 are all equal to $m$ and we have defined $Q_{m}=$ $e^{-m}$, similarly the length of the horizontal lines is $T_{a}$ and we have defined $Q_{a}=e^{-T_{a}}$ such that $t_{a}=T_{a}+m$ is the distance between the two vertical lines. $\lambda, \mu$ and $\eta$ are Young diagrams and $s_{\lambda / \mu}\left(x_{1}, x_{2}, \ldots\right)$ is the skew-Schur polynomial in the variables $x_{1}, x_{2}, \ldots . t^{-\rho}$ denotes the following set of variables: $t^{-\rho}=\left\{t^{1 / 2}, t^{3 / 2}, \ldots\right\}$.

Using repeatedly the identity

$\sum_{\lambda} s_{\lambda^{t} / \eta}(x) s_{\lambda / \sigma}(y)=\prod_{i, j}\left(1+x_{i} y_{j}\right) \sum_{\tau} s_{\sigma^{t} / \tau}(x) s_{\eta^{t} / \tau^{t}}(y)$

and the following properties of the skew-Schur functions:

$$
\begin{aligned}
& s_{\lambda^{t} / \sigma^{t}}\left(q^{-\rho}\right)=s_{\lambda / \sigma}\left(-q^{\rho}\right), \\
& \sum_{\eta} s_{\lambda / \eta}(x) s_{\eta / \sigma}(y)=s_{\lambda / \sigma}(x, y),
\end{aligned}
$$

the partition function in Eq. (3.1) can be written as

$$
\begin{aligned}
Z_{N}= & \Pi\left(Q_{m}\right)^{N} \sum_{\lambda, \tau} Q_{\rho}^{\left|\lambda_{(0)}\right|} \\
& \times \prod_{a=0}^{N-1} s_{\lambda_{(a)} / \tau_{(a+1)}}\left(\mathbf{x}_{a+1}\right) s_{\lambda_{(a+1)} / \tau_{(a+1)}}\left(\mathbf{y}_{a+1}\right),
\end{aligned}
$$

where

$$
\begin{aligned}
Q_{\rho} & =\prod_{a=0}^{N-1}\left(Q_{a} Q_{m}\right), \quad Q_{1, a+1}=\left(Q_{1} Q_{2} \ldots Q_{a}\right) Q_{m}^{a} \\
\mathbf{x}_{a+1} & =Q_{m}^{-1 / 2} Q_{1, a+1}\left\{Q_{m} \sqrt{\frac{q}{t}} t^{\rho}, t^{-\rho}\right\}, \\
\mathbf{y}_{a+1} & =Q_{m}^{-1 / 2} Q_{1, a+1}^{-1}\left\{Q_{m} \sqrt{\frac{t}{q}} q^{-\rho}, q^{\rho}\right\},
\end{aligned}
$$


and

$\Pi(x)=\prod_{i, j=1}^{\infty}\left(1-x q^{-\rho_{i}} t^{-\rho_{j}}\right)$.

In the above equations $-\log \left(Q_{\rho}\right)$ is the Kähler parameter associated to the elliptic curve which is dual to the circle transverse to the M5-branes. The form of the partition function given in Eq. (3.5) was obtained in [4] where it was related to the periodic Schur process of period $N$.

\subsection{Product representation of partition function using free} fermions

In this section we express the partition function given in Eq. (3.5) as an infinite product. To do this, following [5] we introduce the free fermion Fock space spanned by creation and annihilation operators $\left(\psi_{a}, \psi_{a}^{*}\right)$ satisfying the relations

$$
\left\{\psi_{a}, \psi_{b}\right\}=\left\{\psi_{a}^{*}, \psi_{b}^{*}\right\}=0, \quad\left\{\psi_{a}, \psi_{b}^{*}\right\}=\delta_{a b}, a, b \in \mathbb{Z}+\frac{1}{2} .
$$

With these we can construct the operators

$$
\begin{aligned}
\Gamma_{ \pm}(z)=\exp \left(\sum_{n=1}^{\infty} \frac{z^{n} J_{n}}{n}\right), \quad \text { where } \\
J_{n}=\sum_{k \in \mathbb{Z}+\frac{1}{2}} \psi_{k+n} \psi_{k}^{*}, \quad n= \pm 1, \pm 2, \ldots
\end{aligned}
$$

These operators satisfy the following commutation relation:

$\Gamma_{+}(z) \Gamma_{-}(w)=(1-z w) \Gamma_{-}(w) \Gamma_{+}(z)$.

The fermionic Fock space is spanned by states which are in a one-to-one correspondence with partitions. For a partition $\lambda=\left(\lambda_{1}, \lambda_{2}, \ldots\right)$ we have the state

$|\lambda\rangle=\psi_{\lambda_{1}-\frac{1}{2}} \psi_{\lambda_{2}-\frac{3}{2}} \psi_{\lambda_{3}-\frac{5}{2}} \ldots|0\rangle$.

The ground state $|0\rangle$ is annihilated by $\psi_{k}$ for $k<0$. The operators defined in Eq. (3.8) are useful when working with symmetric polynomials due to the following:

$$
\begin{aligned}
\prod_{i} \Gamma_{+}\left(x_{i}\right)|\lambda\rangle & =\sum_{\mu} s_{\mu / \lambda}\left(x_{1}, x_{2}, \ldots\right)|\mu\rangle \\
\prod_{i} \Gamma_{-}\left(x_{i}\right)|\lambda\rangle & =\sum_{\mu} s_{\lambda / \mu}\left(x_{1}, x_{2}, \ldots\right)|\mu\rangle .
\end{aligned}
$$

To simplify the equations we will use the following notation: $\prod_{i} \Gamma_{ \pm}\left(x_{i}\right)=\Gamma_{ \pm}(\mathbf{x})$. Using Eqs. (3.5) and (3.11) we can write $\widehat{Z}_{N}:=Z_{N} / \Pi\left(Q_{m}\right)^{N}$ as a trace, over the free fermionic Fock space, of an infinite product of operators:

$$
\widehat{Z}_{N}=\operatorname{Tr}\left(\left(Q_{1} Q_{m}\right)^{L_{0}} \mathcal{O}\left(Q_{2} Q_{m}\right)^{L_{0}} \mathcal{O} \ldots\left(Q_{N} Q_{m}\right)^{L_{0}} \mathcal{O}\right),
$$

with the operator insertions $\mathcal{O}$ being built from $\Gamma_{ \pm}$,

$$
\begin{aligned}
\mathcal{O}= & \prod_{i=1}^{\infty}\left(\Gamma_{+}\left(Q_{m}^{-1} t^{-i+\frac{1}{2}}\right) \Gamma_{+}\left(\sqrt{\frac{q}{t}} t^{i-\frac{1}{2}}\right)\right. \\
& \times \prod_{j=1}^{\infty}\left(\Gamma_{-}\left(q^{j-\frac{1}{2}}\right) \Gamma_{-}\left(Q_{m} \sqrt{\frac{t}{q}} q^{-j+\frac{1}{2}}\right)\right) .
\end{aligned}
$$

In the above equations $L_{0}$ is such that $L_{0}|\lambda\rangle=|\lambda||\lambda\rangle$. Using the commutation relations of $\Gamma_{ \pm}(\mathbf{x})$, we can write Eq. (3.12) as

$$
\begin{aligned}
\widehat{Z}_{N}=\operatorname{Tr} & \left(Q_{\rho}^{L_{0}} \prod_{a=1}^{k} \Gamma_{+}\left(Q_{1} Q_{2} \ldots Q_{a} Q_{m}^{a} \mathbf{x}\right)\right. \\
& \left.\times \Gamma_{-}\left(Q_{1}^{-1} Q_{2}^{-1} \ldots Q_{a}^{-1} Q_{m}^{-a} \mathbf{y}\right)\right),
\end{aligned}
$$

where

$\mathbf{x}=\left\{Q_{m}^{-1} t^{-i+\frac{1}{2}}, \sqrt{\frac{q}{t}} t^{i-\frac{1}{2}} \mid i=1,2, \ldots\right\}$,
$\mathbf{y}=\left\{q^{i-\frac{1}{2}}, Q_{m} \sqrt{\frac{t}{q}} q^{-i+\frac{1}{2}} \mid i=1,2, \ldots\right\}$.

Using the commutation relation of $\Gamma_{ \pm}(x)$ repeatedly we get

$\widehat{Z}_{N}=\left(\prod_{1 \leq a<b \leq k} F_{a b}\right) \operatorname{Tr}\left(Q_{\rho}^{L_{0}} \Gamma_{+}(\mathbf{X}) \Gamma_{-}(\mathbf{Y})\right)$,

where we have denoted

$$
\begin{aligned}
& \mathbf{x}=\left\{Q_{1} Q_{m} \mathbf{x}, Q_{1} Q_{2} Q_{m}^{2} \mathbf{x}, \ldots, Q_{1} Q_{2} \ldots Q_{N} Q_{m}^{N} \mathbf{x}\right\}, \\
& \mathbf{Y}=\left\{Q_{1}^{-1} Q_{m}^{-1} \mathbf{y}, Q_{1}^{-1} Q_{2}^{-1} Q_{m}^{-2} \mathbf{y}, \ldots, Q_{1}^{-1} Q_{2}^{-1} \ldots Q_{N}^{-1} Q_{m}^{-N} \mathbf{y}\right\},
\end{aligned}
$$

$F_{a b}=\prod_{i, j=1}^{\infty} \frac{\left(1-Q_{a b} Q_{m}^{-1} t^{i-\frac{1}{2}} q^{j-\frac{1}{2}}\right)\left(1-Q_{a b} Q_{m} t^{i-\frac{1}{2}} q^{j-\frac{1}{2}}\right)}{\left(1-Q_{a b} t^{i} q^{j-1}\right)\left(1-Q_{a b} t^{i-1} q^{j}\right)}$,

$Q_{a b}=Q_{a+1} \ldots Q_{b} Q_{m}^{b-a}$.

The trace appearing in Eq. (3.16) can be written in the form of a product [6],

$\operatorname{Tr}\left(Q_{\rho}^{L_{0}} \Gamma_{+}(\mathbf{x}) \Gamma_{-}(\mathbf{y})\right)=\prod_{n=1}^{\infty}\left(1-Q_{\rho}^{n}\right)^{-1} \prod_{i, j}\left(1-Q_{\rho}^{n} x_{i} y_{j}\right)^{-1}$. 
such that the partition function is given by

$\widehat{Z}_{N}=\left(\prod_{n=1}^{\infty}\left(1-Q_{\rho}^{n}\right)^{-1}\right)\left(\prod_{1 \leq a<b \leq N} F_{a b}\right)\left(\prod_{a, b=1}^{N} H_{a b}\right)$,

where

$$
\begin{aligned}
& H_{a b}=\prod_{n, i, j=1}^{\infty} \frac{\left(1-Q_{\rho}^{n} \widetilde{Q}_{a b} Q_{m}^{-1} t^{i-\frac{1}{2}} q^{j-\frac{1}{2}}\right)\left(1-Q_{\rho}^{n} \widetilde{Q}_{a b} Q_{m} t^{i-\frac{1}{2}} q^{j-\frac{1}{2}}\right)}{\left(1-Q_{\rho}^{n} \widetilde{Q}_{a b} t^{i} q^{j-1}\right)\left(1-Q_{\rho}^{n} \widetilde{Q}_{a b} t^{i-1} q^{j}\right)} \\
& \widetilde{Q}_{a b}=Q_{1} Q_{2} \ldots Q_{a} Q_{1}^{-1} \ldots Q_{b}^{-1} Q_{m}^{a-b} .
\end{aligned}
$$

\section{Free energy and Gopakumar-Vafa invariants}

Using the refined topological string partition function we can calculate the free energy and the Gopakumar-Vafa invariants $[7,8]$ for the different curve classes. The free energy is given by

$$
\begin{aligned}
F_{N}\left(\rho, t_{1}, \ldots, t_{N-1}, m\right) & =\ln Z_{N} \\
& =N \ln \Pi\left(Q_{m}\right)+\ln \widehat{Z}_{N},
\end{aligned}
$$

where $\Pi\left(Q_{m}\right)$ is given by Eq. (3.6) and $\widehat{Z}_{N}$ is given by Eq. (3.22). After some simplification $F_{N}$ can be written as

$$
\begin{aligned}
& F_{N}\left(\rho, t_{1}, \ldots, t_{N-1}, m\right) \\
& =-N \sum_{k=1}^{\infty} \frac{Q_{m}^{k}}{k}\left[\frac{1}{\left(q^{\frac{k}{2}}-q^{-\frac{k}{2}}\right)\left(t^{\frac{k}{2}}-t^{-\frac{k}{2}}\right)}\right]+\sum_{n=1}^{\infty} \sum_{k=1}^{\infty} \frac{Q_{\rho}^{n k}}{k} \\
& \quad+\sum_{1 \leq a<b \leq N} \sum_{k=1}^{\infty} \frac{Q_{a b}^{k}}{k}\left[\frac{(t / q)^{\frac{k}{2}}+(q / t)^{\frac{k}{2}}-\left(Q_{m}^{k}+Q_{m}^{-k}\right)}{\left(q^{\frac{k}{2}}-q^{-\frac{k}{2}}\right)\left(t^{\frac{k}{2}}-t^{-\frac{k}{2}}\right)}\right] \\
& \quad+\sum_{n=1}^{\infty} \sum_{a, b=1}^{N} \sum_{k=1}^{\infty} \frac{Q_{\rho}^{n k} \widetilde{Q}_{a b}^{k}}{k}\left[\frac{(t / q)^{\frac{k}{2}}+(q / t)^{\frac{k}{2}}-\left(Q_{m}^{k}+Q_{m}^{-k}\right)}{\left(q^{\frac{k}{2}}-q^{-\frac{k}{2}}\right)\left(t^{\frac{k}{2}}-t^{-\frac{k}{2}}\right)}\right] .
\end{aligned}
$$

From Eq. (4.2) we can see that the $S U(2)_{L} \times S U(2)_{R}$ spin content of various curve classes is given by

\begin{tabular}{ll}
\hline Curve & $\sum_{j_{L}, j_{R}} N_{C}^{j_{L}, j_{R}}\left(j_{L}, j_{R}\right)$ \\
\hline$n E+C_{a b}, \quad n \geq 0,1 \leq a<b \leq$ & $\left(0, \frac{1}{2}\right)$ \\
$\quad N, c=a-1, b+1$ & $(0,0)$ \\
$n E+C_{a b}+M_{c}, \quad n \geq 0,1 \leq a<b \leq$ & \\
$\quad N, c=a-1, b+1$ & $(0,0)$ \\
$n E+C_{a b}-M_{c}, \quad n \geq 0,1 \leq a<b \leq$ & \\
$\quad N, c=a+1, b-1$ & $\left(0, \frac{1}{2}\right)$ \\
$n E-C_{a b}, \quad n \geq 1,1 \leq a<b \leq N$ & $(0,0)$ \\
$n E-C_{a b}+M_{c}, \quad n \geq 1,1 \leq a<b \leq$ & $(0,0)$ \\
$\quad N, c=a-1, b+1$ & \\
$n E-C_{a b}-M_{c}, \quad n \geq 1,1 \leq a<b \leq$ & $\left(\frac{1}{2}, 0\right) \oplus(N-1)\left(0, \frac{1}{2}\right)$ \\
$\quad N, c=a+1, b-1$ & \\
$n E, \quad n \geq 1$ &
\end{tabular}

Here

$C_{a b}=C_{a}+C_{a+1}+\cdots+C_{b}$.

From Eq. (4.2) we can isolate the contribution of the elliptic curve $E$ to $F_{N}$, which we will denote by $F_{N}^{E}$, by considering only those terms which depend only on $Q_{\rho}$ and the other Kähler parameter,

$F_{N}^{E}(\rho, q, t)=\sum_{n, k=1}^{\infty} \frac{Q_{\rho}^{n k}}{k}\left[1+N\left(\frac{t^{k}+q^{k}}{\left(1-q^{k}\right)\left(1-t^{k}\right)}\right)\right]$.

We will restrict ourselves to the unrefined case so that $q=t$ and in terms of the topological string coupling constant $g_{s}$,

$q=t=e^{i g_{s}}$.

Since

$\frac{2 q}{(1-q)^{2}}=\frac{2}{g_{s}^{2}}-\frac{1}{6}-2 \sum_{g \geq 2}^{\infty} g_{s}^{2 g-2} \frac{B_{2 g}}{(2 g)(2 g-2) !}$,

where $B_{k}$ are the Bernoulli numbers, we have

$F_{N}^{E}(\rho, q)=\sum_{g=0}^{\infty} g_{s}^{2 g-2} F_{N, g}^{E}(\rho)$

with

$$
\begin{aligned}
F_{N, g \geq 2}^{E}= & N \frac{B_{2 g} B_{2 g-2}}{2(2 g)(2 g-2) !} \\
& -2 N \frac{B_{2 g}}{(2 g)(2 g-2) !} \frac{B_{2 g-2}}{4} E_{2 g-2}(\rho), \\
F_{1}^{E}= & -\frac{1}{12} \ln \left[\prod_{n=1}^{\infty}\left(1-Q_{\rho}^{n}\right)^{12-2 N}\right] \\
& \frac{\partial^{2} F_{0}^{E}}{\partial \rho^{2}}=2 N \sum_{n=1}^{\infty} n^{2} \ln \left(1-Q_{\rho}^{n}\right)^{-1}
\end{aligned}
$$

where $E_{2 g}(\rho)$ is the Eisenstein series defined by

$$
E_{2 g}(\rho)=1-\frac{4}{B_{2 g}} \sum_{n \geq 1} n^{2 g-1}\left(\frac{Q_{\rho}^{n}}{1-Q_{\rho}^{n}}\right)
$$

\section{Non-perturbative modular transformation}

For an elliptic Calabi-Yau threefold with modular parameter $\rho$ one naively would expect the partition function to be invariant under the modular transformation,

$Z_{N}\left(-\frac{1}{\tau}, \frac{\epsilon_{1}}{\tau}, \frac{\epsilon_{2}}{\tau}\right)=Z_{N}\left(\tau, \epsilon_{1}, \epsilon_{2}\right)$

where $q=e^{i \epsilon_{1}}$ and $t=e^{-i \epsilon_{2}}$. However, quite surprisingly, in [1] it was shown that the refined topological partition function 
of $X_{1}$, which is dual to a single M5-brane wrapped on a circle, satisfies a non-perturbative modular transformation,

$Z_{1}\left(-\frac{1}{\tau}, \frac{\epsilon_{1}}{\tau}, \frac{\epsilon_{2}}{\tau}\right)=\frac{Z_{1}\left(\tau, \epsilon_{1}, \epsilon_{2}\right)}{Z_{1}\left(\frac{\tau}{\epsilon_{1}},-\frac{1}{\epsilon_{1}}, \frac{\epsilon_{2}}{\epsilon_{1}}\right) Z_{1}\left(\frac{\tau}{\epsilon_{2}}, \frac{\epsilon_{1}}{\epsilon_{2}},-\frac{1}{\epsilon_{2}}\right)}$.

Since $\epsilon_{1}, \epsilon_{2}$ are related to the topological string coupling constant $g_{s}$ by

$\epsilon_{1} \epsilon_{2}=-g_{s}^{2}$,

Equation (5.2) implies that

$Z_{1}\left(-\frac{1}{\tau}, \frac{\epsilon_{1}}{\tau}, \frac{\epsilon_{2}}{\tau}\right)=Z_{1}\left(\tau, \epsilon_{1}, \epsilon_{2}\right)+O\left(e^{-\frac{1}{g s}}\right)$,

i.e., modularity holds only up to non-perturbative corrections. For this reason the transformation in Eq. (5.2) was called a non-perturbative modular transformation and it was argued that this gives the non-perturbative completion of the topological string partition function for the case of elliptic Calabi-Yau threefolds.

To show that $Z_{N}$ also satisfies an equation similar to Eq. (5.2) we write it in terms of double elliptic gamma functions. Recall that the double elliptic Gamma function is defined by

$$
\begin{aligned}
G_{2}\left(x ; \tau, \epsilon_{1}, \epsilon_{2}\right)= & \prod_{k, i, j=1}^{\infty}\left(1-Q_{\tau}^{k-1} q^{i-1} t^{-j+1} x\right) \\
& \times\left(1-Q_{\tau}^{k} q^{i} t^{-j} x^{-1}\right)
\end{aligned}
$$

and satisfies the following modular transformation:

$$
\begin{aligned}
& G_{2}\left(z ; \rho, \epsilon_{1}, \epsilon_{2}\right) \\
& =G_{2}\left(\frac{z}{\rho} ;-\frac{1}{\rho}, \frac{\epsilon_{1}}{\rho}, \frac{\epsilon_{2}}{\rho}\right) G_{2}\left(\frac{z}{\epsilon_{1}} ; \frac{\rho}{\epsilon_{1}},-\frac{1}{\epsilon_{1}}, \frac{\epsilon_{2}}{\epsilon_{1}}\right) \\
& \quad \times G_{2}\left(\frac{z}{\epsilon_{2}} ; \frac{\rho}{\epsilon_{2}}, \frac{\epsilon_{1}}{\epsilon_{2}},-\frac{1}{\epsilon_{2}}\right) \exp \left(\frac{i \pi}{12} B_{44}\right),
\end{aligned}
$$

where $B_{4,4}$ is given by

$$
B_{4,4}\left(z ; \rho, \epsilon_{1}, \epsilon_{2}\right)=\left.\frac{\mathrm{d}^{4}}{\mathrm{~d} x^{4}} \frac{x^{4} e^{z x}}{\left(e^{\rho x}-1\right)\left(e^{\epsilon_{1} x}-1\right)\left(e^{\epsilon_{2} x}-1\right)}\right|_{x=0} .
$$

The triple infinite product in Eq. (3.23) can be written in terms of double elliptic gamma functions so that the full partition function $Z_{N}$ is given by

$$
\begin{aligned}
Z_{N} & =Z_{1}^{N}\left(\prod_{n=1}^{\infty}\left(1-Q_{\rho}^{n}\right)\right)^{N-1} \\
& \times \prod_{1 \leq a<b \leq N} \frac{G_{2}\left(Q_{a b} Q_{m} \sqrt{t q} ; \rho, \epsilon_{1},-\epsilon_{2}\right) G_{2}\left(Q_{a b} Q_{m}^{-1} \sqrt{t q} ; \rho, \epsilon_{1},-\epsilon_{2}\right)}{G_{2}\left(Q_{a b} t ; \rho, \epsilon_{1},-\epsilon_{2}\right) G_{2}\left(Q_{a b} q ; \rho, \epsilon_{1},-\epsilon_{2}\right)} \\
= & Z_{1}^{N}\left(\prod_{n=1}^{\infty}\left(1-Q_{\rho}^{n}\right)\right)^{N-1} \\
& \times \prod_{1 \leq a<b \leq N} \frac{G_{2}\left(Q_{a b} ; \rho, \epsilon_{1}, \epsilon_{2}\right) G_{2}\left(Q_{\rho} Q_{a b}^{-1} ; \tau, \epsilon_{1}, \epsilon_{2}\right)}{G_{2}\left(Q_{a b} Q_{m} \sqrt{t q} ; \rho, \epsilon_{1}, \epsilon_{2}\right) G_{2}\left(Q_{a b} Q_{m}^{-1} \sqrt{t q} ; \rho, \epsilon_{1}, \epsilon_{2}\right)}
\end{aligned}
$$

with the explicit expression

$$
\begin{aligned}
Z_{1}= & \left(\prod_{n=1}^{\infty}\left(1-Q_{\rho}^{n}\right)^{-1}\right) \\
& \times \prod_{i, j=1}^{\infty} \frac{\left(1-Q_{\rho}^{n-1} Q_{m} q^{i-\frac{1}{2}} t^{j-\frac{1}{2}}\right)\left(1-Q_{\rho}^{n} Q_{m}^{-1} q^{i-\frac{1}{2}} t^{j-\frac{1}{2}}\right)}{\left(1-Q_{\rho}^{n} q^{i} t^{j-1}\right)\left(1-Q_{\rho}^{n} q^{i-1} t^{j}\right)} .
\end{aligned}
$$

Using Eq. (5.8) and the modular transformation satisfied by $G_{2}\left(z ; \rho, \epsilon_{1}, \epsilon_{2}\right)$ we see that

$$
\frac{Z_{N}\left(\tau, \epsilon_{1}, \epsilon_{2}\right)}{Z_{N}\left(\frac{\tau}{\epsilon_{1}},-\frac{1}{\epsilon_{1}}, \frac{\epsilon_{2}}{\epsilon_{1}}\right) Z_{N}\left(\frac{\tau}{\epsilon_{2}}, \frac{\epsilon_{1}}{\epsilon_{2}},-\frac{1}{\epsilon_{2}}\right)}=Z_{N}\left(-\frac{1}{\tau}, \frac{\epsilon_{1}}{\tau}, \frac{\epsilon_{2}}{\tau}\right) .
$$

Thus this class of elliptic Calabi-Yau threefolds do indeed satisfy the non-perturbative modular transformation of Lockhart and Vafa [1].

\section{Discussion}

In this paper we have shown that there is a simple class of elliptic Calabi-Yau threefolds for which the product representation of the refined topological string partition function can be determined using the relation between Schur functions and free fermionic Fock space. The product representation allows us to study the non-perturbative modular transformations of [1]. The class of elliptic Calabi-Yau threefolds discussed in this paper were simplest in the sense that they had no compact 4-cycles. It would be interesting to see if the non-perturbative modular transformation holds for an elliptic Calabi-Yau threefold with compact 4-cycles. We hope to report on this in the future [9]. 
Open Access This article is distributed under the terms of the Creative Commons Attribution 4.0 International License (http://creativecomm ons.org/licenses/by/4.0/), which permits unrestricted use, distribution, and reproduction in any medium, provided you give appropriate credit to the original author(s) and the source, provide a link to the Creative Commons license, and indicate if changes were made.

Funded by SCOAP ${ }^{3}$.

\section{References}

1. G. Lockhart, C. Vafa, Superconformal partition functions and nonperturbative topological strings (2012). arXiv:1210.5909 [hep-th]

2. B. Haghighat, A. Iqbal, C. Kozcaz, G. Lockhart, C. Vafa, M-Strings (2015). arXiv:1305.6322 [hep-th]
3. A. Iqbal, C. Kozcaz, C. Vafa, The refined topological vertex. JHEP 0910, 069 (2009). arXiv:hep-th/0701156 [hep-th]

4. A. Iqbal, B.A. Qureshi, K. Shabbir, M.A. Shehper, Brane webs and random processes (2015). arXiv:1509.06295 [hep-th]

5. A. Okounkov, N. Reshetikhin, Correlation function of Schur process with application to local geometry of a random 3-dimensional Young diagram (2003). arXiv:math/0107056 [math.CO]

6. I.G. Macdonald, Symmetric functions and hall polynomials, 2nd edn. (Oxford Science Publications, Oxford Mathematical Monographs, Oxford, 1995)

7. R. Gopakumar, C. Vafa, M theory and topological strings-I (1998). arXiv:hep-th/9809187 [hep-th]

8. R. Gopakumar, C. Vafa, M theory and topological strings-II (1998). arXiv:hep-th/9812127 [hep-th]

9. A. Iqbal, B.A. Qureshi, K. Shabbir, Work in progress (2016) 